\title{
Paillard, Bernard, Simon, Jean-François, Le Gall, Laurent (dir.), En France rurale. Les enquêtes interdisciplinaires depuis les années 1960
}

\section{Aurélie Hess-Miglioretti}

\section{(2) OpenEdition Journals \\ Édition électronique \\ URL : http://journals.openedition.org/abpo/2039 \\ DOI : 10.4000/abpo.2039 \\ ISSN : 2108-6443 \\ Éditeur}

Presses universitaires de Rennes

\section{Édition imprimée}

Date de publication : 30 mai 2011

Pagination : 191-192

ISBN : 978-2-7535-1691-5

ISSN : 0399-0826

Référence électronique

Aurélie Hess-Miglioretti, « Paillard, Bernard, Simon, Jean-François, Le Gall, Laurent (dir.), En France rurale. Les enquêtes interdisciplinaires depuis les années 1960 », Annales de Bretagne et des Pays de l'Ouest [En ligne], 118-2 | 2011, mis en ligne le 30 juin 2013, consulté le 10 décembre 2020. URL http://journals.openedition.org/abpo/2039; DOI : https://doi.org/10.4000/abpo.2039

Ce document a été généré automatiquement le 10 décembre 2020.

(c) Presses universitaires de Rennes 


\title{
Paillard, Bernard, Simon, Jean- François, Le Gall, Laurent (dir.), En France rurale. Les enquêtes interdisciplinaires depuis les années 1960
}

\author{
Aurélie Hess-Miglioretti
}

\section{RÉFÉRENCE}

PAILLARD, Bernard, SIMON, Jean-François, LE GALL, Laurent (dir.), En France rurale. Les enquêtes interdisciplinaires depuis les années 1960, Rennes, PUR, 2010, 396 p.

1 En mai 2008 s'est tenu à Plozévet, à l'initiative de Bernard Paillard, un colloque organisé par le CRBC (Centre de Recherche Bretonne et Celtique) et l'université de Bretagne occidentale consacré aux «Grandes enquêtes pluridisciplinaires des années 1960-70. Bilans et perspectives » et à la recherche coopérative sur programme de Plozévet qui fut menée entre 1961 et 1965 en particulier. Ce colloque a donné lieu à cet ouvrage qui regroupe des témoignages des enquêteurs et des relectures des enquêtes de Plozévet ainsi que des autres enquêtes effectuées des années 1960 aux années 1980 sur certaines portions de l'espace rural français (l'Aubrac, le Châtillonnais, les Baronnies, la Corse).

2 Dans cet ouvrage des enquêteurs de l'époque retracent ce que furent leurs travaux en redéfinissant non seulement le contexte scientifique de ces enquêtes et de leur recrutement, mais aussi leurs méthodes de travail et leurs relations avec les habitants, les autorités et les autres chercheurs. Ils apportent un témoignage sur la place de ces enquêtes dans la recherche et dans leur carrière en particulier. 
Ces grandes enquêtes ont participé à la construction d'un savoir sur les sociétés rurales en pleine transformation. En particulier sur la société rurale bretonne avec les enquêtes de Plozévet. Elles furent les premières enquêtes pluridisciplinaires lancées en France dans les années 1960. Ce livre tente de répondre à deux questions : Pourquoi et comment les enquêtes dans cette petite commune du Finistère? Il cherche aussi à donner quelques pistes de réflexion en montrant l'actualité de ces enquêtes. Des enquêtes de Plozévet on espérait retirer de grandes avancées dans la connaissance globale d'une collectivité rurale contemporaine. En outre, la multidisciplinarité devait déboucher sur l'interdisciplinarité.

Robert Gessain, alors sous-directeur du Musée de l'homme, militait pour l'étude transversale de petites populations et du mécanisme d'homogénéisation de ces populations. La notion d'isolat, concept issu de la génétique des populations, est le fondement de l'étude des petites populations et définit la population comme une unité biologique. Cependant, une commune comme Plozévet est une unité administrative et non une unité biologique. La coïncidence entre la circonscription administrative et l'isolat est un présupposé basé sur le calcul de taux d'endogamie. La commune fut donc observée sous tous les angles comme le produit d'une réalité sociale alors même que les frontières sociales ne sont en aucun cas des frontières administratives. Des distorsions apparaissent dans cette approche territorialisée de phénomènes sociaux (François Ploux).

5 Le nom donné à cette enquête pilote de Plozévet est : l'« étude du monde agricole et rural français dans son adaptation aux conditions de vie modernes ». La spécificité bretonne n'est alors absolument pas privilégiée. Jean Sutter qui travaillait sur la luxation congénitale de la hanche, avait identifié le pays Bigouden comme étant le centre de ce phénomène. Il a donc choisi le département du Finistère. Les archives de Plozévet étaient bien tenues, ce qui permettait des études longitudinales et des études de parenté. Le choix s'est donc porté sur cette petite commune rurale bretonne.

6 L'action concertée de Plozévet a mobilisé une centaine de chercheurs de toutes les disciplines de sciences humaines et sociales: sociologues, géographes, ethnologues, historiens, psychosociologues, anthropologues physiques ont participé à ces enquêtes. De manière à favoriser l'interdisciplinarité, une vie en commun est privilégiée afin d'encourager les contacts et les discussions entre les enquêteurs des disciplines représentées. Cette recommandation ne fut cependant guère respectée. Solange PetitSkinner, chargée de la coordination entre les chercheurs, témoigne même d'un manque de coopération et d'une méfiance entre eux. Les travaux de recherche sont donc restés largement cloisonnés entre les disciplines, la recherche n'est devenue en aucun cas un modèle de recherche interdisciplinaire.

7 Ces recherches ont donné lieu à une quarantaine de rapports, cinq livres et de nombreux articles. En parallèle de ces enquêtes Robert Gessain et sa femme ont réalisé cinq films ethnographiques. Par ailleurs, Christian Pelras a réalisé un carnet d'enquête, filmé lors de sa recherche ethnologique menée à Goulien en même temps que les enquêtes de Plozévet. Il étudiait une commune du Cap Sizun plus homogène, à la population moins nombreuse et plus représentative. Cette étude devait permettre une comparaison avec la population de Plozévet.

8 Les ruralistes, ou sociologues ruraux, sont cependant absents de ces enquêtes alors même que l'agriculture française est en pleine mutation avec un exode rural important 
et que la définition de la politique agricole commune est au cœur des préoccupations du monde rural.

De ces enquêtes restent aussi les rumeurs et la polémique entre chercheurs et plozévétiens qui se sont côtoyés sans toujours se comprendre. Cependant aucune suite n'a été donnée aux enquêtes de Plozévet dont la synthèse tardive par A. Burguière (Bretons de Plozévet, Paris, Flammarion, 1975) a été publiée seulement en 1975. Les recherches collectives qui ont suivi ont tiré les leçons de ces premières enquêtes mais ont toujours tenu à s'en différencier. L'enquête a été contestée au niveau local où une approche trop superficielle et souvent parisianiste été dénoncée. Néanmoins, Masahiro Ogino évoque ce qu'un chercheur japonais peut retirer de cette expérience française d'enquête in vivo. Il revient sur l'influence au Japon des travaux d'Édgar Morin à Plozévet. Le recours à la convivialité doit réduire la distance entre enquêteur et enquêté.

Pour Jean François Simon, ces enquêtes ont joué un grand rôle dans l'élaboration de l'ethnologie de la Bretagne. Il s'interroge à travers l'expérience de Plozévet sur la manière dont les Bretons ont développé une ethnologie de la Bretagne. Il revient sur le rôle de Donatien Laurent, un breton habitant Paris mais qui souhaitait travailler sur la Bretagne et notamment sur la musique bretonne et celtique.

11 Ces enquêtes, comme les autres recherches collectives, ont permis le développement de nouvelles problématiques, le renforcement des équipes et ont constitué le vivier de recrutement de jeunes chercheurs.

12 L'ouvrage fait aussi un retour sur l'organisation institutionnelle de la recherche en France à partir du début des années 1960 avec un zoom sur l'état de la recherche en sciences humaines et sociales de l'époque. Il existait une différence entre les disciplines académiques constituées de longue date comme l'histoire et la géographie et des disciplines plus récentes en voie de reconnaissance académique comme la sociologie, la psychologie ou l'ethnologie.

13 L'ensemble des témoignages des enquêteurs de l'époque montre que l'analyse des archives de ces enquêtes est un véritable élément non seulement de l'histoire des sciences sociales, mais aussi de la pluridisciplinarité. Elles apportent de nouvelles pistes de réflexion. En 50 ans, les thèmes de recherche, les méthodes d'enquête et de travail ainsi que les rapports entre les disciplines ont beaucoup évolué. Est-ce que l'histoire des enquêtes reste à faire? C'est certain pour Bernard Paillard qui souhaite les replacer dans l'histoire de chacune des disciplines. Les enquêtes de Plozévet et de Goulien ont acquis une densité historique, elles marquent l'histoire des sciences humaines et sociales en Bretagne. 